\title{
Clinical anatomy of the spatial structure of the right ventricular outflow tract
}

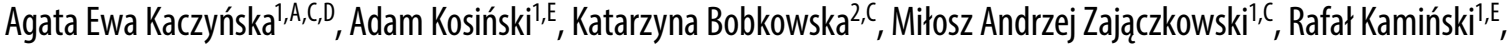 \\ Grzegorz Marek Piwko ${ }^{1, E}$, Marta Gleinert-Rożek ${ }^{1, B}$, Tomasz Gos ${ }^{3, B}$, Karol Karnecki, ${ }^{3, B}$, Dariusz Kozłowski ${ }^{4, F}$ \\ ${ }^{1}$ Department of Clinical Anatomy, Medical University of Gdańsk, Poland \\ 2 Faculty of Civil and Environmental Engineering, Gdańsk University of Technology, Poland \\ ${ }^{3}$ Department of Forensic Medicine, Medical University of Gdańsk, Poland \\ ${ }^{4}$ Department of Cardiology and Electrotherapy, Medical University of Gdańsk, Poland \\ A - research concept and design; B - collection and/or assembly of data; $\mathrm{C}$ - data analysis and interpretation; \\ $D$ - writing the article; $E$ - critical revision of the article; $F$ - final approval of the article
}

Address for correspondence

Adam Kosińnki

adam.kosinski@gumed.edu.pl

Funding sources

This work was supported by the National

Science Centre, grant No. MN-01-0412/08/212

(http://www.nauka.gov.pl/en/).

Conflict of interest

None declared

Received on 0ctober 27, 2020

Reviewed on November 11, 2020

Accepted on December 17, 2020

Published online on November 5, 2021

\section{Abstract}

Background. The right ventricular outflow tract (RVOT) is located above the supraventricular crest and reaches the level of the pulmonary valve. Detailed knowledge of the RVOT spatial structure and its morphology is extremely important for cardiac invasive therapeutic procedures.

Objectives. To examine the spatial structure of the RVOT using virtual models of the right ventricle (RV) interior obtained post mortem.

Materials and methods. The study was carried out using 40 adult hearts from both sexes fixed in formalin. Donors had a negative history of cardiovascular diseases. Silicone models of the interior of the RV were made and then subjected to a digital modelling procedure using the photogrammetry technique. For each 3D model of the RV, the RVOT was extracted and measurements were performed.

Results. Statistical analysis demonstrated that the dimensions of the transverse $(p<0.001)$ and sagittal $(p=0.002)$ axis at the level of the upper and lower border of the RVOT differed significantly. There was also a significant difference between the right and left height of the RVOT $(p=0.009)$. A clear correlation was found between the volume of the RVOT and the volume of the entire RV ( $r=0.718, p<0.001)$.

Conclusions. The obtained 3D models of the RVOT can help standardize the data related to RVOT architecture. Furthermore, they can extend knowledge about the RVOT in the field of cardiology and improve the procedures in cardiac surgery.

Key words: photogrammetry, RVOT, right ventricle, cardiac imaging, 3D modelling

Cite as

Kaczyńska AE, Kosińnki A, Bobkowska K, et al. Clinical anatomy of the spatial structure of the right ventricular outflow tract. Adv Clin Exp Med. 2022;31(1):33-40. doi:10.17219/acem/131752

DOI

10.17219/acem/131752

Copyright

Copyright by Author(s)

This is an article distributed under the terms of the

Creative Commons Attribution 3.0 Unported (CC BY 3.0)

(https://creativecommons.org/licenses/by/3.0/) 


\section{Background}

The right ventricle (RV) and structures located in its area are gradually gaining more interest among researchers. The relationship between anatomical components of the RV and various heart diseases is being increasingly recognized. There are reports in the scientific literature related to the analysis of the structure and function of the RV, both for healthy hearts and those with pathological changes. ${ }^{1,2}$ There are also many papers that present the spatial architecture of the RV and its components. One notable study is that by Ho and Nihoyannopoulos, who comprehensively described the RV and its internal structures in terms of echocardiography. ${ }^{1}$ Unfortunately, much of the available data about the context of the nomenclature of the structures within the RV are still ambiguous.

One component of the RV sparking serious controversies is the right ventricular outflow tract (RVOT), which is the outflow path of the blood flowing through the RV during the cardiac cycle. The RVOT area is located between the pulmonary valve and the level of the upper part of the supraventricular crest. ${ }^{1,2}$

Due to its clinical significance, the RVOT is an important region for morphological studies. It has been shown that the knowledge of the shape of this area is of great importance in the context of the implantation of cardiac stimulating systems. ${ }^{3}$ Furthermore, the source of idiopathic ventricular arrhythmias is often located within the RVOT. ${ }^{4}$ Therefore, thorough knowledge of this area and the structures located in its cavity is the basis for optimizing ablation procedures for arrhythmogenic foci presented by Pytkowski et al. ${ }^{5}$

From a practical perspective, the spatial structure of the RVOT seems to be particularly important. Information obtained from 3D modelling can serve as a source of valuable and abundant data, especially in clinical aspects. Due to modern developments in imaging techniques, cardiac visualization is becoming an essential component of the diagnostic and therapeutic process in cardiology and cardiac surgery.

Photogrammetry, which has been used in medicine for many years, is a technique that enables imaging of anatomical structures post mortem. In the context of the heart, photogrammetric methods are primarily aimed at understanding the shape and size of this organ. Due to the ability to make silicone models of the heart cavities, it has also become possible to analyze these spaces. An additional application and major advantage of photogrammetric techniques is the ability to obtain a durable 3D model that can be processed and analyzed many times. Photogrammetry is most often associated with the use of the visible electromagnetic radiation range (which was used for the purpose of image registration in this study), although other ranges of radiation are also used, e.g. x-rays, near and mid-infrared radiation, etc.

The importance of photogrammetry in medicine has significantly increased and it is now associated with the computerization and digitization of many medical analysis processes.
For example, it can be used to model the requirements for 3D printing of missing or replacement structures ${ }^{6}$ or for the collection of objective data about the dimensions and shapes of given organs (as in the present study). A dataset collected using photogrammetry can be used for many years.

\section{Objectives}

The aim of this study was to perform manual measurements of RVOT dimensions and volumes based on virtual models of the RV interior obtained post mortem in order to analyze RVOT morphology.

\section{Materials and methods}

\section{Model preparation}

Observations were carried out for 40 hearts from adults aged 18-85 years (average age: 44 years) of both sexes (13 women, 27 men). All donors had a negative history of cardiovascular diseases. The hearts were fixed in formalin solution. In order to obtain the RVOT model, 40 silicone internal models of the RV were first made according to the procedure described in Kaczyńska et al. ${ }^{7}$ (Fig. 1).

This study was approved by the Independent Bioethics Committee for Scientific Research at Medical University of Gdańsk, Poland (approval No. 407/2015 granted on September 15, 2015).

\section{Digital modelling}

The obtained models were subjected to a digital modelling procedure using photogrammetry techniques. This method uses serial photos of the physical model to transform it into a virtual model. For this purpose, a Sony Alpha DSLR-A300 (Sony Corp., Tokyo, Japan) camera was used with a Sony 3.5-5.6/18-70 lens (Sony Corp.), and 50 continuous photos were taken of each model. Next, the photos were imported into Autodesk ReCap Photo (Autodesk Inc., San Rafael, USA), transformed into a virtual model, and then scaled. In addition, digital processing and manual RVOT measurements were made (Fig. 2).

\section{Right ventricular outflow tract boundary determination}

Before manual processing of the 3D models, macroscopic analyses of the obtained silicone models and specimens of the interior of the RV were carried out to determine the characteristic points that would be used to evaluate the RVOT boundaries. During the process of extracting the RVOT from the 3D model of the entire RV, a virtual vertical cut was first made, starting from the location of the supraventricular crest - the largest indentation on the model 


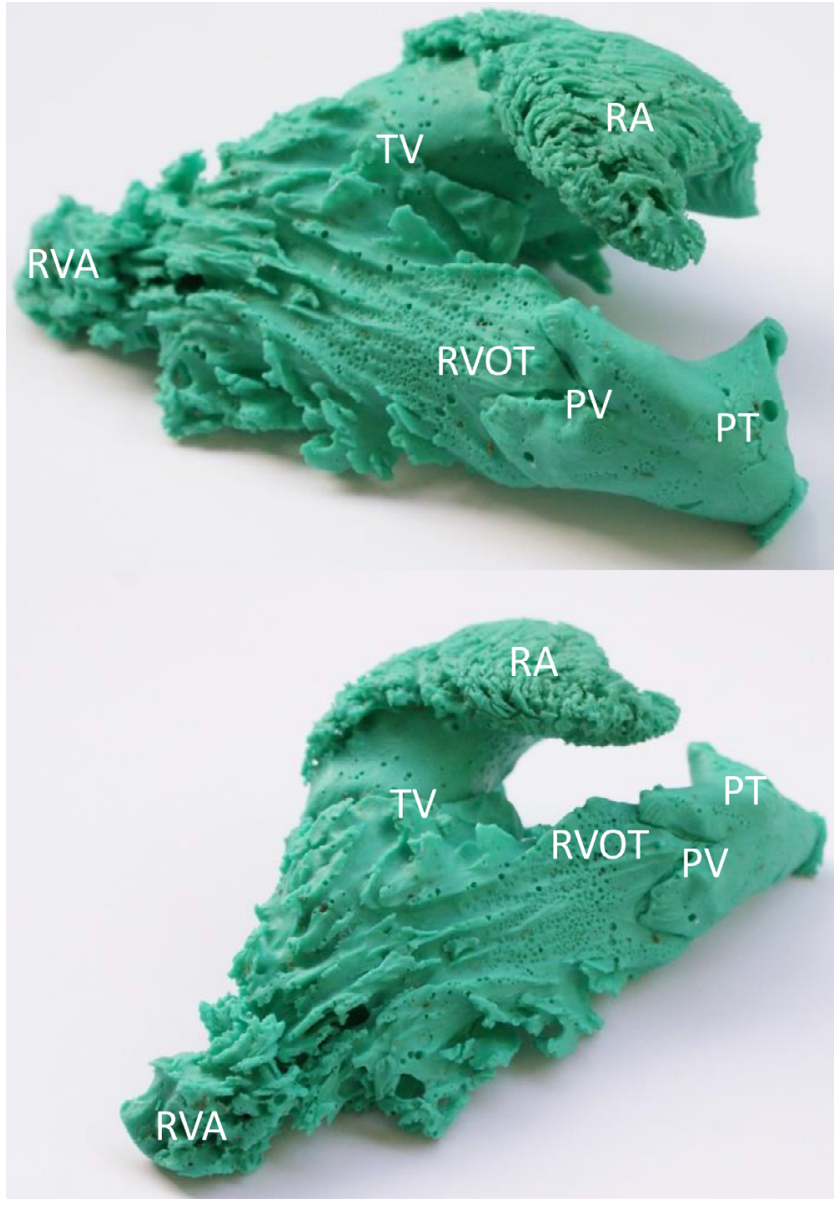

Fig. 1. Silicone model of the interior

RA - right atrium; TV - tricuspid valve; RVA - right ventricle apex; RVOT - right ventricle outflow tract; PT - pulmonary trunk; PV - pulmonary valve.

- to the apex of the RV. Next, from the first of these points, another cut was made to the left edge of the RV, where the free wall meets the septal wall. On this edge, the location of the first characteristic indentation on the model (from the pulmonary valve to the apex of the RV) was determined, which defines the lower border of the RVOT. Hearts were grouped according to the presence of indentation or no visible indentation. The upper border of the RVOT was defined as the ring connecting the attachment points (commissural points) of the pulmonary valve cusps (Fig. 3A).

\section{Manual measurements}

For the obtained 40 models of the RVOT, the following measurements of the spatial structures were performed: the largest dimension in the transverse axis, at the level of the upper (a) and lower (a') border of the RVOT; the largest dimension in the sagittal axis, at the level of the upper (b) and lower (b') border of the RVOT and the right (c) and left (c') height of the RVOT, connecting the furthest points at the upper and lower border of the RVOT (Fig. 4). The RVOT volume and the volume of the entire RV were also measured prior to extracting the RVOT.

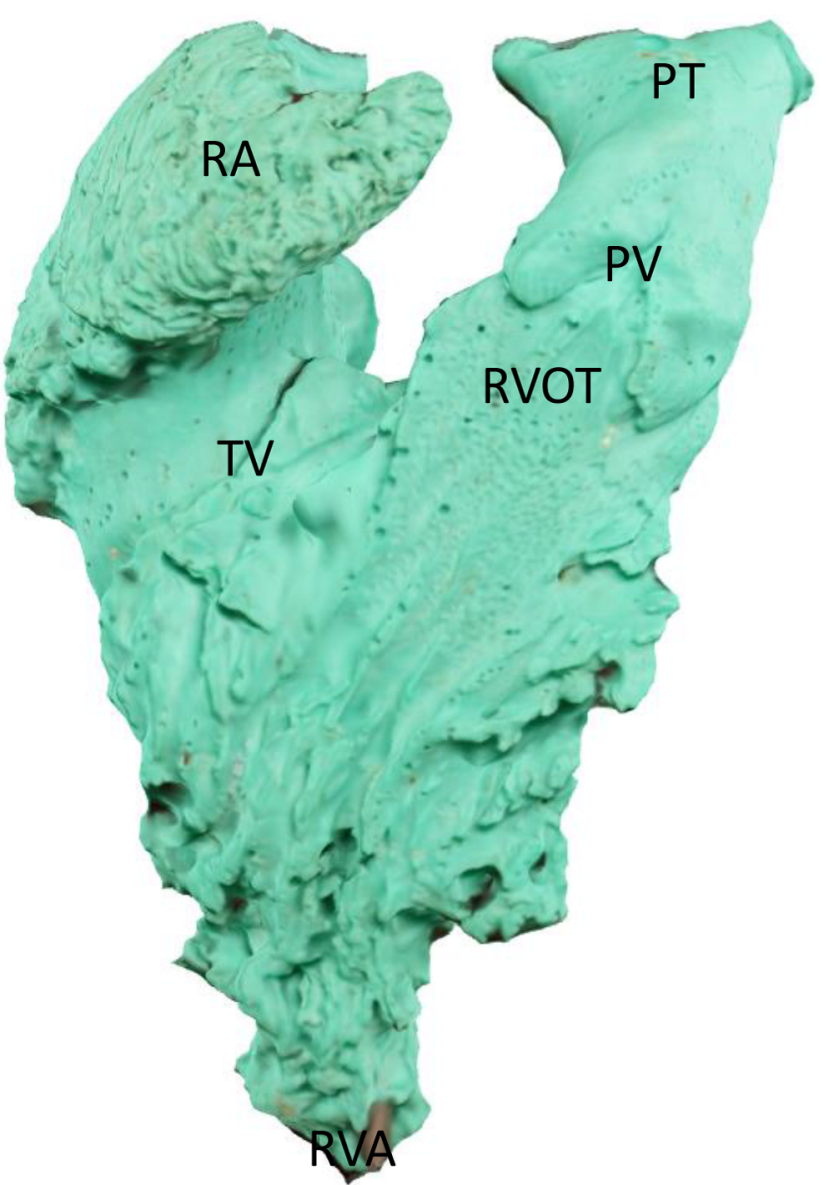

Fig. 2. Digitized silicone model

RA - right atrium; TV - tricuspid valve; RVA - right ventricle apex; RVOT - right ventricle outflow tract; PT - pulmonary trunk: $\mathrm{PV}$ - pulmonary valve.

\section{Statistical analysis}

Statistical analysis was performed using Statistica Software v. 13.1 for Windows (StatSoft Inc., Tulsa, USA). The D'Agostino's and Pearson's tests were used to check the normality of the distribution. The data had a normal distribution. Student's t-test for independent samples was used to compare the RVOT measurements. This test was chosen because of the small sample size ( 40 hearts) and for comparing 2 groups. Pearson's correlation analysis was performed to assess the relationship between the entire RV volume and the RVOT volume. Results with $\mathrm{p}<0.05$ were considered statistically significant.

\section{Results}

\section{Macroscopic analysis}

The RVOT was extracted from each of the forty 3D models of the RV. During the determination of the RVOT boundaries, 2 characteristic indentations were observed on the virtual models. The $1^{\text {st }}$ indentation was 


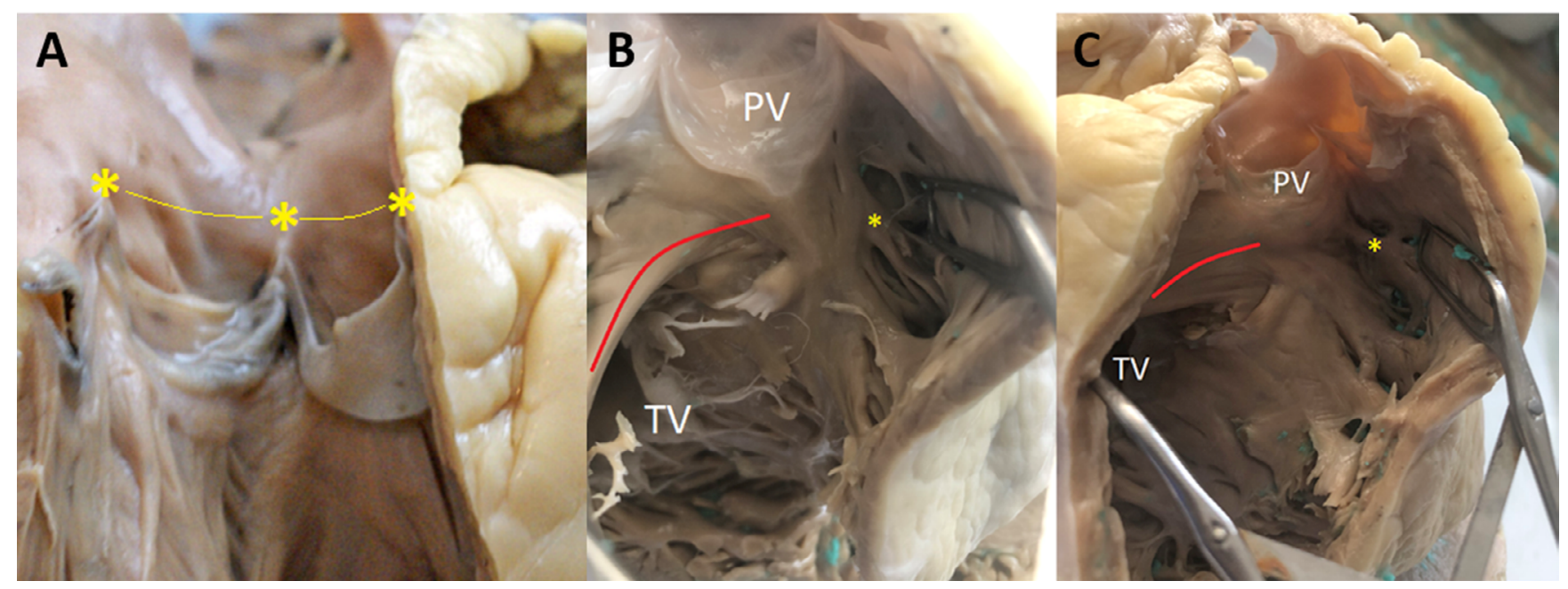

Fig. 3. A. Setting the upper border of the right ventricle outflow tract (RVOT). The yellow asterisk indicates the attachments of the pulmonary valve cusps to the internal surface of the vessel. The line marks are the assumed upper border of the RVOT; B and C. The view of the RVOT from the perspective of the free wall. The yellow asterisk indicates the extension of the supraventricular crest that causes characteristic indentation on the 3D model. The red line marks the supraventricular crest that separates the inflow from outflow tract in the right ventricle (RV)

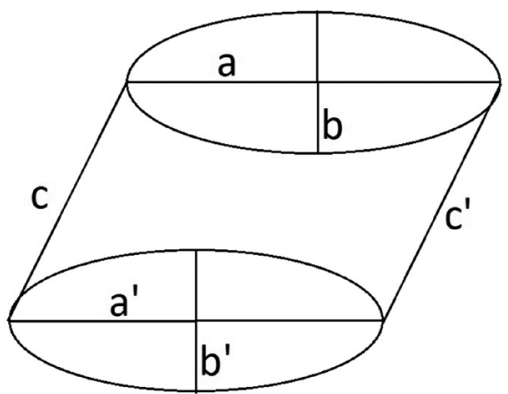

Fig. 4. Scheme of the measurements performed for the right ventricle outflow tract (RVOT)

a - dimension in the transverse axis at the level of the upper border of the RVOT; $a^{\prime}$ - dimension in the transverse axis at the level of the lower border of the RVOT; $b$ - dimension in the sagittal axis at the level of the upper border of the RVOT; $b^{\prime}$ - dimension in the sagittal axis at the level of the lower border of the RVOT; $c$ - right border of RVOT; $c^{\prime}$ - left border of RVOT.

the equivalent of the supraventricular crest that separated the RVOT on the model from the right atrioventricular orifice. The lowest point lying on this indentation was used to determine the virtual cut towards the apex and left margin of the RV. The $2^{\text {nd }}$ indentation was on the left margin of the RV and was located as the highest point towards the pulmonary valve. It was used to set the $2^{\text {nd }}$ point in order to determine the lower border of the RVOT (Fig. 5).

A characteristic indentation was not present only on 4 RVOT models or it was not possible to identify it (Fig. 5A). The rest 36 RVOT models had a noticeable indentation (Fig. 5B,C). After indentation analysis of the 3D models, macroscopic assessment of this structure was carried out on the specimens fixed in formalin. It was found that the structure that created the characteristic indentation on the 3D model during the process of silicone modelling is a muscular element, that connects the septal wall with the free wall of the RV (Fig. 3B). This structure emerges below the pulmonary valve cusps, at the level of the supraventricular crest, and then goes downwards overlapping the free wall of the RV. Above this element, the trabeculation is less intense compared to the RV architecture below this structure. The macroscopic examination also revealed that the protruding element does not reach the point where the septal wall connects directly with the free wall of the RV. The structure appeared as a free bundle of muscles connecting both walls. Deriving a line along the supraventricular crest and the examined structure, it was found that the line along the examined element is an extension of the supraventricular crest, and both marked lines form an arch that curves below the pulmonary valve (Fig. 3C).

\section{Right ventricular outflow tract dimensions and volume}

The mean dimension in the transverse axis of the RVOT at the level of the upper border (a) was $23.51 \mathrm{~mm}$ (standard deviation $(\mathrm{SD})=4.81 \mathrm{~mm}$ ); the same mean dimension at the level of the lower border (a') was $28.18 \mathrm{~mm}$ $(\mathrm{SD}=6.36 \mathrm{~mm})$. Statistical analysis showed that the dimension in the transverse axis differed significantly at the level of the upper and lower RVOT (Student's t-test, $\mathrm{p}<0.001$; Fig. 6).

The obtained data indicated that this dimension, starting from the lower border to the upper one, became smaller. The mean dimension in the sagittal axis at the level of the upper border of the structure (b) was $18.24 \mathrm{~mm}$ $(\mathrm{SD}=4.85 \mathrm{~mm})$, while that at the level of the lower border (b') was $14.95 \mathrm{~mm}(\mathrm{SD}=4.56 \mathrm{~mm})$. This difference was statistically significant (Student's t-test, $\mathrm{p}=0.002$ ), indicating that the anteroposterior dimension of the RVOT becomes larger towards the pulmonary valve (Fig. 7). 

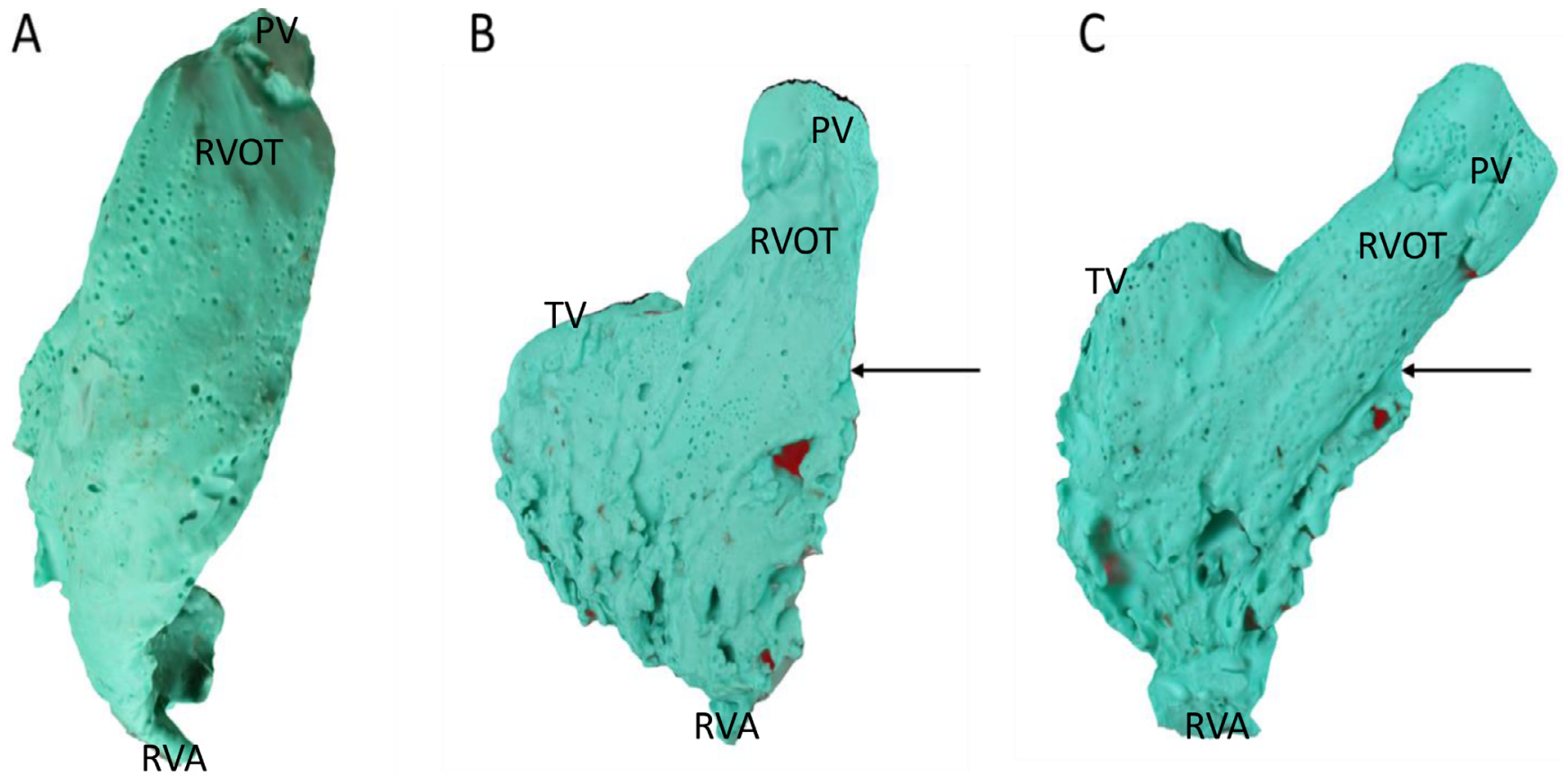

Fig. 5. A view of the 3D model of the right ventricle (RV). A. No visible indentation; B and C. Visible indentation (marked with an arrow)

TV - tricuspid valve; RVA - right ventricle apex; RVOT - right ventricle outflow tract; PV - pulmonary valve.

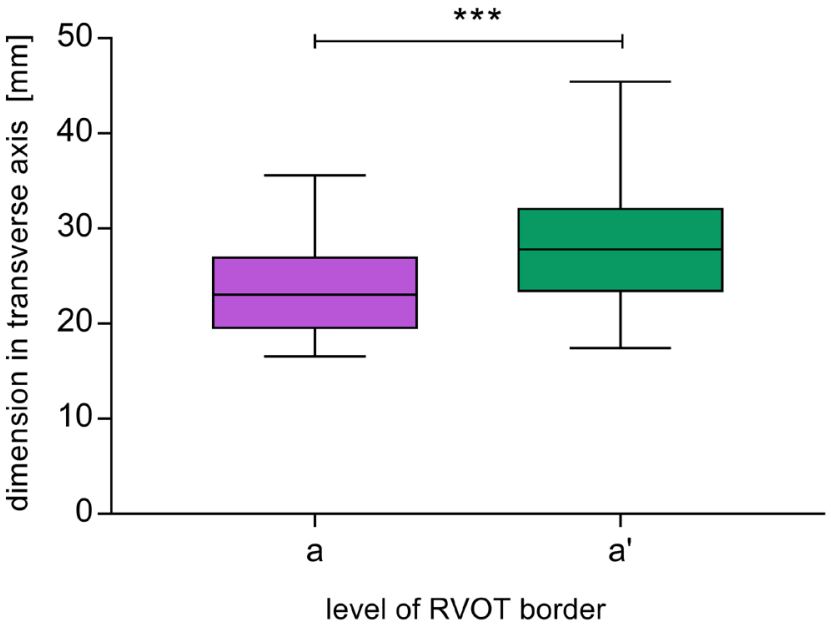

Fig. 6. Comparison of the dimension in the transverse axis at the level of the upper (a) and lower ( $a^{\prime}$ ) border of the right ventricle outflow tract (RVOT). The values are given in millimeters [mm]

$\mathrm{a}-\mathrm{M}=23.51$, standard deviation $(\mathrm{SD})=4.81 ; \mathrm{a}^{\prime}-\mathrm{M}=28.18, \mathrm{SD}=6.36$.

The obtained data also allowed us to assess the right (c) and left (c') mean height of the RVOT structure, which was $35.96 \mathrm{~mm}(\mathrm{SD}=6.02 \mathrm{~mm})$ and $39.45 \mathrm{~mm}(\mathrm{SD}=5.63 \mathrm{~mm})$, respectively. The difference between these measurements was statistically significant (Student's t-test, $\mathrm{p}=0.009$ ) (Fig. 8). The left height (c') of the RVOT was significantly greater than the right height (c).

The mean volume of the RVOT was $11.98 \mathrm{~mL}$, which comprised $24 \%$ of the volume of the entire RV. There was a strong positive correlation between the volume of the RVOT and the volume of the entire RV $(\mathrm{r}=0.718, \mathrm{p}<0.001)$. The obtained data clearly show that

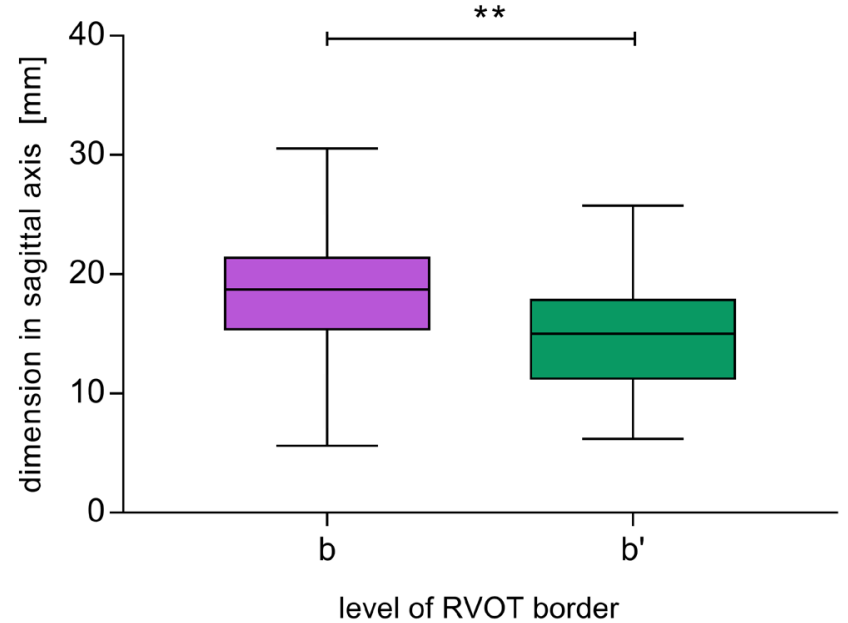

Fig. 7. Comparison of the dimension in the sagittal axis at the level of the upper (b) and lower ( $b^{\prime}$ ) border of the right ventricle outflow tract (RVOT). The values are given in millimeters [mm]

$b-M=18.24$, standard deviation $(S D)=4.85 ; b^{\prime}-M=14.95, S D=4.56$.

as the volume of the entire RV increases, the RVOT volume also increases (Fig. 9).

\section{Discussion}

At present, the interior of the RV is divided into 3 parts: inflow tract, trabeculated apex and outflow tract. However, the existing data lack the definition of the exact border between the RVOT and the trabeculated apex of the RV. Due to conflicting data, knowledge about this area is inconsistent. 


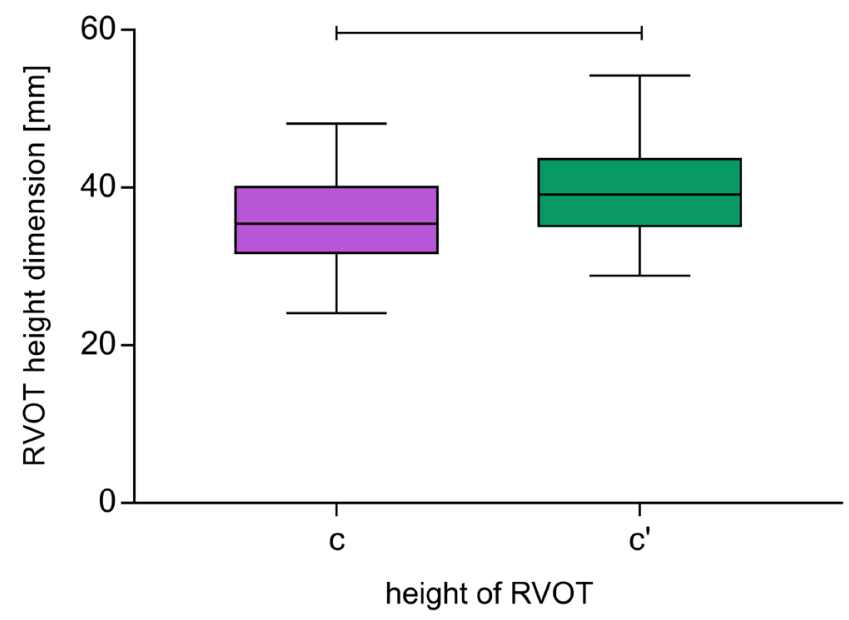

Fig. 8. Comparison of the right (c) and left ( $c^{\prime}$ ) border of the right ventricle outflow tract (RVOT). The values are given in millimeters [mm]

$C-M=35.96$, standard deviation $(S D)=6.02 ; c^{\prime}-M=39.45, S D=5.63$.

The supraventricular crest, which separates the inflow tract from outflow tract of the RV, forms a noticeable boundary structure between the 2 components of the RV. This structure penetrates the interventricular septum between the anterior and posterior limb of the septomarginal trabecula.

The pulmonary infundibulum, which is part of the RVOT, has the form of a smooth and muscular sleeve. Along that structure extends the anterior limb of the septomarginal trabecula, which supports the cusps of the pulmonary valve by lifting the entire valve apparatus above the base of the heart. This is possible because the fragments of the infundibulum are built into the 3 pulmonary sinuses. The locations of the attachments of the pulmonary valve cusps determine the sinotubular junction, while the anatomical border between the RV and the pulmonary trunk is defined as the line to which the infundibular musculature reaches within the base of the cusps sinuses. ${ }^{1,8,9}$ Despite large variation in length, size and angle of the curvature of the pulmonary infundibulum, its dimensions do not depend on the dimensions of the RV itself. ${ }^{10}$

In the present study, the place of attachment of the pulmonary valve cusps to the inner surface of the vessel wall was assumed to be the upper border of the RVOT. The connected attachment points of the pulmonary valve formed a ring that was easy to locate on the $3 \mathrm{D}$ models of the RV. Determining the lower RVOT border was unfortunately not as easy, due to the lack of a clear border between the trabecular apex and the infundibulum..$^{8,11-13}$

Based on our observations, the structure most suitable for determining the lower border seems to be the element of the RV that causes the characteristic indentation on the 3D model in the area of contact between the free and septal wall. This indentation occurred in up to $90 \%$ of the examined hearts, and may thus serve as a determinant of the lower border of the RVOT. Macroscopic analysis of the specimens revealed that the indentation

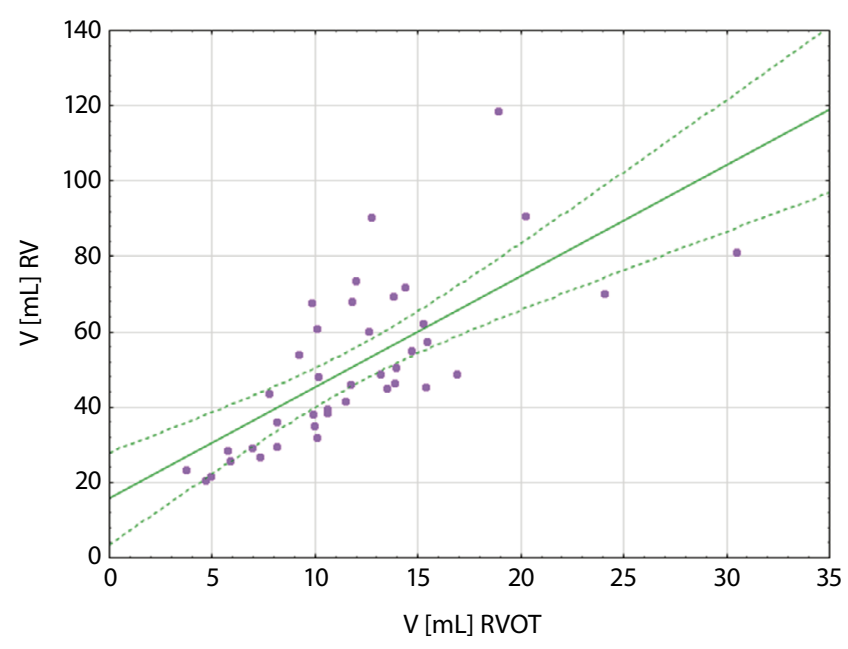

Fig. 9. Correlation between the volume of the entire right ventricle (V RV) and the volume of the right ventricle outflow tract (V RVOT). The values are given in milliliters $[\mathrm{mL}]$

is caused by a muscle structure that passes from the septal wall to the free wall, directly from the pulmonary trunk to the apex of the RV (Fig. 9). Notably, this element is the last protrusion towards the infundibulum that has a smooth wall. Moreover, this structure appears as a free muscle bundle that attaches to the septal wall. Due to its connection with the free and septal walls, it can be determined that this element is not a typical part of the septoparietal trabeculation that lies on the internal surface of the walls of the RV. This structure is also not a free part of the septomarginal trabecula, as evidenced by its too high location in the ventricular space. Based on macroscopic analysis of this structure, it seems to be an extension of the supraventricular crest on the free wall of the RV. In a study by Kosiński et al., the trabecular element was described as a component of the RV.${ }^{14}$ It was an extension of the ventricular septal muscle bundle and was indicated as the lower part of the supraventricular crest. Reaching the base of the heart, this element separated the inflow tract from the RVOT, which was visible in our macroscopic analysis of the specimens. It seems that the macroscopic characteristics of the structure that causes the protrusion on the 3D models corresponds to the extended part of the supraventricular crest.

Assuming that this structure is a part of the supraventricular crest, it seems justified to use it as a determinant of the lower border of RVOT, because it occurs in the vast majority of cases. Importantly, its location within the RV is permanent, compared to the variable trabeculation. In addition, the supraventricular crest has an analogous embryological origin to the RVOT. ${ }^{15,16}$

Previous scientific reports have attempted to establish the lower border of RVOT, which was typically determined in the context of the implantation of cardiac pacing or ablation of arrhythmogenic foci. In a study by Mond et al., ${ }^{17}$ the trabecular part was included in the RVOT area and the lower border of the RVOT was designated as the line 
from the highest point of the tricuspid valve towards the left edge of the heart. Based on its different electrophysiological properties, the RVOT was divided into 4 wall segments: anterior, posterior, free wall part, and ventricular septum.

In order to define the optimal pacing sites in the RVOT, Lieberman et al. similarly marked the lower border of this area. ${ }^{3}$ The placement of an electrode in a specific segment of the RVOT is characterized by a different morphology of the QRS complex in electrocardiography. Thus, unifying the definition of the RVOT anatomical boundaries is crucial for various cardiac procedures.

In contrast to the findings of Saremi et al. ${ }^{8}$ that the size of the infundibulum, which is an element of the RVOT, is not dependent on the size of the entire RV, our results showed that the volume of the RVOT is dependent on the volume of the entire RV. Thus, it can be concluded that as the RV volume of the examined hearts increases, the RVOT volume also increases. However, the specific dimensions, angles and spatial structures of the RVOT and RV may differ significantly and they require further research.

In a study by Izumo et al. using 3D transesophageal echocardiography (3D TEE), it was shown that the RVOT cross-sectional geometry is more oval than round, as previously thought. ${ }^{18}$ The fact that we set the lines connecting the points placed furthest apart from each other in the sagittal and transverse axis, at the level of the upper and lower RVOT border, also supports this view. In addition, we showed that the transverse dimension becomes smaller towards the upper border of the RVOT while the sagittal dimension increases. The different values of these dimensions certainly reflect the change in the shape of the RVOT cross section. For this reason, it is necessary to carefully examine these dimensions at different levels of the RVOT sections. In addition, differences in the left and right height of the RVOT structure indicate a change in the position of its subsequent sections and their certain displacement, which may be associated with RVOT rotation.

A study by Bobkowska et al. reported that differences in measurements between physical and virtual models are negligible. ${ }^{19}$ The developed modelling procedure is characterized by intuitiveness, ease of implementation by people less experienced in photogrammetry, and good quality results. The experiments carried out by the authors indicate the possibility of obtaining better quality models than those obtained using optical scanners. ${ }^{19}$ Manual photo recording allows any orientation and adjustment of the camera distance to the object. The significant advantages of this method justified its choice to digitize silicone models of the RV interior in the present study.

\section{Clinical implications}

Extension of knowledge about the anatomy of the RVOT using imaging and modelling techniques may contribute to the optimization of pacemaker implantation procedures, resulting in more effective cardiac stimulation, better fixation of the stimulating lead, and less frequent mechanical interaction of the device with the valvular apparatus. In patients with arrhythmias within the RVOT area, better understanding of RVOT morphology may help to more accurately localize the arrhythmogenic foci, thereby increasing the effectiveness of ablation treatment. Moreover, it may be helpful in avoiding ablation-related damage to structures located in close proximity to the RVOT.

\section{Limitations}

This research, however, is subject to several limitations. First, the study was carried out on relatively small number of hearts and thus the RVOTs, which is related to the availability of specimens.

Another limitation is the disproportion between the number of hearts of both sexes, with male hearts being the majority.

Additionally, the density of the silicone used in the models preparation may be insufficient for modeling the smallest structures inside RV.

\section{Conclusions}

Post mortem 3D modelling of the RV interior allows for a comprehensive analysis of RVOT anatomy. We demonstrated that the RVOT diameter narrows towards the pulmonary trunk; RVOT height is not symmetrical, which may be associated with RVOT rotation; and RVOT volume is proportionate to total RV volume.

Further studies are needed to collect more data on morphological variants of the RVOT, especially studies based on the analysis of cross sections.

\section{ORCID iDs}

Agata Ewa Kaczyńska (D) https://orcid.org/0000-0002-7509-5839 Adam Kosiński (D) https://orcid.org/0000-0001-7507-2285 Katarzyna Bobkowska (D) https://orcid.org/0000-0003-4968-3407 Miłosz Andrzej Zajączkowski (D) https://orcid.org/0000-0002-5981-336X Rafał Kamiński (D) https://orcid.org/0000-0003-3632-0340 Grzegorz Marek Piwko (D) https://orcid.org/0000-0003-2301-6075 Marta Gleinert-Rożek (D) https://orcid.org/0000-0001-7807-4323 Tomasz Gos (D) https://orcid.org/0000-0003-0161-9270

Karol Karnecki (i) https://orcid.org/0000-0001-8386-4207

Dariusz Kozłowski (D) https://orcid.org/0000-0003-2089-0656

\section{References}

1. Ho SY, Nihoyannopoulos P. Anatomy, echocardiography, and normal right ventricular dimensions. Heart. 2006;92(Suppl 1):i2-13. doi:10. 1136/hrt.2005.077875

2. Sanz J, Sánchez-Quintana D, Bossone E, Bogaard HJ, Naeije R. Anatomy, function, and dysfunction of the right ventricle: JACC state-ofthe-art review. J Am Coll Cardiol. 2019;73(12):1463-1482. doi:10.1016/j. jacc.2018.12.076

3. Lieberman R, Grenz D, Mond HG, Gammage MD. Selective site pacing: Defining and reaching the selected site. Pacing Clin Electrophysiol. 2004;27(6 Pt 2):883-886. doi:10.1111/j.1540-8159.2004.00551.x 
4. Kukla P, Sławuta A, Jastrzębski M, Gajek J, Stec S. Unusual changes in ventricular repolarization before right ventricular outflow tract arrhythmias. Am J Med Sci. 2017;353(3):311-312. doi:10.1016/j.amjms. 2016.11.009

5. Pytkowski M, Maciąg A, Sterliński M, et al. Lokalizacja ogniska arytmii u chorych z zaburzeniami rytmu serca pochodzącymi z drogi odpływu prawej komory. Folia Cardiol Excerpta. 2006;1(4):211-220. https://journals.viamedica.pl/folia_cardiologica/article/view/24007. Accessed September 7, 2021.

6. Liaw $C Y$, Guvendiren M. Current and emerging applications of 3D printing in medicine. Biofabrication. 2017;9(2):024102. doi:10.1088/ 1758-5090/aa7279

7. Kaczyńska A, Kosiński A, Kamiński R, Zajączkowski M, Nowicka E, Gleinert-Rożek M. A novel approach to visualization of the right ventricular outflow tract. Eur J Trans/ Clin Med. 2019;1(2):36-40. doi:10. 31373/ejtcm/100603

8. Saremi F, Ho SY, Cabrera JA, Sánchez-Quintana D. Right ventricular outflow tract imaging with CT and MRI: Part 1, morphology. Am J Roentgenol. 2013;200(1):W39-W50. doi:10.2214/AJR.12.9333

9. Loukas M, Shane Tubbs R, Louis RG, et al. An endoscopic and anatomical approach to the septal papillary muscle of the conus. Surg Radiol Anat. 2009;31(9):701-706. doi:10.1007/s00276-009-0510-2

10. Geva T, Powell AJ, Crawford EC, Chung T, Colan SD. Evaluation of regional differences in right ventricular systolic function by acoustic quantification echocardiography and cine magnetic resonance imaging. Circulation. 1998;98(4):339-345. doi:10.1161/01.cir.98.4.339

11. Keren A, Billingham ME, Popp RL. Echocardiographic recognition of paraseptal structures. J Am Coll Cardiol. 1985;6(4):913-919. doi:10. 1016/s0735-1097(85)80505-2
12. Merrick AF, Yacoub MH, Ho SY, Anderson RH. Anatomy of the muscular subpulmonary infundibulum with regard to the Ross procedure. Ann Thorac Surg. 2000;69(2):556-561. doi:10.1016/s0003-4975(99) 01300-4

13. Hasdemir C, Aktas S, Govsa F, et al. Demonstration of ventricular myocardial extensions into the pulmonary artery and aorta beyond the ventriculo-arterial junction. Pacing Clin Electrophysiol. 2007;30(4): 534-539. doi:10.1111/j.1540-8159.2007.00704.x

14. Kosiński A, Nowiński J, Kozłowski D, Piwko G, Kuta W, Grzybiak M. The crista supraventricularis in the human heart and its role in the morphogenesis of the septomarginal trabecula. Ann Anat. 2007; 189(5):447-456. doi:10.1016/j.aanat.2007.01.008

15. Anderson RH, Webb S, Brown NA, Lamers W, Moorman A. Development of the heart: (3) Formation of the ventricular outflow tracts, arterial valves, and intrapericardial arterial trunks. Heart. 2003;89(9): 1110-1118. doi:10.1136/heart.89.9.1110

16. Poelmann RE, Mikawa T, Gittenberger-De Groot AC. Neural crest cells in outflow tract septation of the embryonic chicken heart: Differentiation and apoptosis. Dev Dyn. 1998;212(3):373-384. doi:10.1002/ (SICI)1097-0177(199807)212:3<373::AID-AJA5>3.0.CO;2-E

17. Mond HG, Hillock RJ, Stevenson IH, McGavigan AD. The right ventricular outflow tract: The road to septal pacing. Pacing Clin Electrophysiol. 2007;30(4):482-491. doi:10.1111/j.1540-8159.2007.00697.x

18. Izumo M, Shiota M, Saitoh T, et al. Non-circular shape of right ventricular outflow tract a real-time 3-dimensional transesophageal echocardiography study. Circ Cardiovasc Imaging. 2012;5(5):621-627. doi:10.1161/CIRCIMAGING.112.974287

19. Bobkowska K, Przyborski M, Kaczyńska A, Kosiński A. Digital photogrammetry in the analysis of the ventricles' shape and size. Paper presented at: 2017 Baltic Geodetic Congress (BGC Geomatics); 2017; Gdańsk, Poland. 